\title{
Knowledge, Attitude and Practice on Common Diabetic Patients among Diabetic Complications at Egyptian Hospital in Mogadishu, Somalia
}

\author{
Mohamed Hassan Mohamed ${ }^{(10)}$, Maryan Ahmed Farah ${ }^{2}$, Mohamed Abdulkadir Ali3, \\ Najma Abdirahman Mohamed33, Abdiqafar Mohamed Hassan ${ }^{3}$ \\ ${ }^{1}$ Department of Public Health, College of Health Science, Somali International University, Mogadishu, Somalia \\ ${ }^{2}$ Somali International University, Mogadishu, Somalia \\ ${ }^{3}$ Faculty of Medicine and Surgery, Somali International University, Mogadishu, Somalia \\ Email: tacshiir@gmail.com,maryankarie01@gmail.com,Drehamada@gmail.com,neeroson0152@gmail.com
}

How to cite this paper: Mohamed, M.H., Farah, M.A., Ali, M.A., Mohamed, N.A. and Hassan, A.M. (2021) Knowledge, Attitude and Practice on Common Diabetic Patients among Diabetic Complications at Egyptian Hospital in Mogadishu, Somalia. Journal of Biosciences and Medicines, 9, 87-99. https://doi.org/10.4236/jbm.2021.99008

Received: October 5, 2020

Accepted: September 7, 2021

Published: September 10, 2021

Copyright $\odot 2021$ by author(s) and Scientific Research Publishing Inc. This work is licensed under the Creative Commons Attribution International License (CC BY 4.0).

http://creativecommons.org/licenses/by/4.0/

(c) (i) Open Access

\begin{abstract}
Diabetes is a chronic illness that occurs when the pancreas stops producing insulin or when the body cannot use the produced insulin. Insulin is a hormone produced by the pancreas that helps glucose from meals enters the body's cells for energy. The overall goal is to assess diabetes patients' knowledge, attitudes, and behaviors about prevalent diabetic complications. This research used a descriptive qualitative methodology to assess diabetes complication knowledge, attitude, and practice. Ninety-eight diabetic patients were visiting the Egyptian Hospital in Mogadishu for four weeks. The data was collected using questionnaires. Finally, SPSS 20 was utilized for analysis. The research was performed from May to July 2020 with a non-probability sample size of 98 diabetics. The bulk of responders were aged $40-60$ (36.7\%). Type 2 was the most common (47.96\%). $44.9 \%$ of respondents had diabetes for more than ten years. $60.2 \%$ had regular checkups, and 54.1 percent had therapy. $52 \%$ of participants were on a diet. $63.3 \%$ of respondents do not exercise often. However, most responders know the common issues (75\%). Less than half of those polled had problems (41.8\%). 22\% incidence of Diabetic ketoacidosis. $78.6 \%$ of respondents know variables that decrease diabetes complications, whereas $22.4 \%$ do not. $37.8 \%$ of respondents saw a doctor for diabetes problems, whereas $62.2 \%$ did not. Diabetic patients' knowledge, attitude, and practice were satisfactory for fundamental illness entanglements and self-testing. These findings may also be due to patient notification and, therefore, long-term illness that leads them to get acquainted with things alone. However, the tendency for regular exercise was low, which may produce more
\end{abstract}


specific consequences of illness. Conclusion: We find that DM patients' KAP was adequate in terms of knowledge of diabetic complications. These results may explain the patients' long-standing illness, which forces them to discover things independently. At the same time, just a handful of them were familiar with vasculopathy and cardiovascular disorders. However, the researchers discovered low level of attitudes and practice toward regular exercise and insufficient levels of attitudes and practice toward diet management and treatment adherence. In general, this degree of KAP is suboptimal and requires further assessment of KAP obstacles in Somalia.

\section{Keywords}

Blood Sugar Level, Diabetes Complications, Diabetes Mellitus, Diabetic Patient, Knowledge

\section{Introduction}

Insulin is a hormone made by the pancreas that demonstrations like a key to let glucose from the food we eat go from the circulatory system into the cells in the body to deliver energy. All sugar nourishments are separated into glucose in the blood. Insulin assists glucose with getting into the cells. Not having the option to create insulin or use it successfully prompts brought glucose step up in the blood [1].

Over the drawn-out high glucose levels are related to harm to the body and disappointment of different organs and tissues (International Diabetes Federation). Hyperglycemia makes harms numerous tissues in the body, prompting the advancement of handicapping and hazardous unexpected issues including loss of vision, kidney disappointment, coronary episodes, strokes, leg removals, and cardiovascular breakdown [2].

Diabetes caused 1.5 million passing in 2012. Higher-than-ideal glucose caused a further 2.2 million passing by expanding the dangers of cardiovascular and different ailments. 43 percent of those 3.7 million passing happens before the age of 70 years. The portion of passing because of high glucose or diabetes that occurs before age 70 is higher in low-and center salary nations than in high-pay countries [3].

In Africa, Diabetes is one of the premier basic ongoing conditions brutal narratives. It's a genuine general wellbeing concern around the world. The movement of diabetes much of the time prompts constant inconveniences, which brings down patients' satisfaction and expands their grimness and mortality. It likewise forces a fantastic monetary weight on wellbeing frameworks [4].

An expected 19.4 million grown-ups matured 20 - 79 years were living with diabetes inside the Africa Region in 2019, speaking to a territorial commonness of $3.9 \%$. Africa is that the Region with the absolute best extent of undiscovered diabetes, with $60 \%$ of grown-ups at present living with diabetes unconscious of 
their condition [5].

Ethiopia is one of the 32 nations of the International Diabetes Federation in the Africa Region. Four hundred fifteen million individuals include diabetes inside the world and more than 14 million people in the Africa Region. By 2040 this figure will be more than double. Concurring International Diabetes Federation, in Africa Region there have been over 1.33 million instances of diabetes and in Ethiopia in 2015 and subsequently, the pervasiveness of diabetes in grown-up is $3 \%[6]$.

In Somalia, Diabetes predominance ( $0 \%$ of populace ages 20 to 79 ) of Somalia increased from $3 \%$ in 2010 to $5.1 \%$ in 2019 growing at an average annual of $42.98 \%$ (IDF). Predictable with the freshest WHO information distributed in 2017, DM Deaths in Somalia arrived at 1019 or $0.81 \%$ of all-out passing. The age-balanced death rate is 22.70 per 100,000 of the populaces. It has been found in past investigations that expanding persistent information concerning DM and its resultant difficulties has benefits in accomplishing understanding consistency in treatment, which can cause abatement in entanglements [7].

\section{Problem Statement}

Diabetes is one of the principal basic non-transmittable maladies of the stylish world, influencing 422 million individuals worldwide and causes an expected 1.5 million passing every year. It's a genuine general well-being concern worldwide with a predominance of $8 \%$ inside us. The movement of diabetes as a rule prompts ongoing entanglement, which brings down patients' satisfaction and expands their horribleness and mortality. It additionally forces a magnificent monetary weight on well-being frameworks frequency of DM is expanding which although there's proof that the difficulties of diabetes are frequently forestalled, there are still patients who come up short on the predetermined information and abilities to oversee and control their condition. DM might be a danger factor for cardiovascular and renal confusion, hyperglycemia makes harms a few tissues inside the body, bringing about the occasion of handicapping and dangerous unexpected problems including loss of vision, renal disappointment, coronary episodes, strokes, leg removals, and coronary disappointment. Steady with the WHO Global Report on Diabetes (2016), the pervasiveness of retinopathy in diabetic customers is $35 \%$. Retinopathy makes $1.9 \%$ of moderate serious vision misfortune [8].

The pervasiveness of End-Stage Renal Disease in a diabetic customer is 12 to $55 \%$. Likewise, the pervasiveness of cardiovascular occasions and lower furthest point removal is 2 to multiple times and 10 to multiple times higher in diabetic patients than the non-diabetic patient's separate. In Somalia, diabetes is thought to be incessant. This investigation will attempt to know the degree of information, perspectives, and practices of diabetes and its normal difficulties at Egyptian hospitals [9].

\section{Knowledge of Diabetic Complication}

Diabetes, everything being equal, can cause intricacies in numerous pieces of the 
body and may expand the overall danger of passing on rashly. Diabetes can influence the vast majority of the body organs particularly the heart, veins, kidneys, eyes, nerves, and teeth [10].

One of the clarifications for event DM difficulty is the absence of information. The basic reasons for diabetic complexities are helpless to control diabetes either because of non-adherence, the helpless mentality towards the illness and its inconveniences, undesirable eating routine, and inadequate physical action, and gratitude to helpless administration by the medical care experts. On the head of that diabetes, difficulties can incline the patient to different diseases [11].

Data about information on the difficulty of diabetic Mellitus is critical for wellbeing arranging. Yet, little is perceived about information about the difficulties of diabetic Mellitus inside the investigation zone. If we alter and improve the information demeanor and practice the occasion of diabetic difficulties will diminish advancement of inconveniences, Studies have indicated that a ton of patients didn't have adequate information on DM entanglements, expanding their insight and mindfulness about such maladies and their complexities will help in lessening the dreariness and mortality of such sicknesses and its intricacies [12].

\subsection{Diabetic Complications}

Eyes: Diabetes raises the chances of getting vision issues, including visual impairment. It can cause Cataracts, Glaucoma, and Retinopathy.

Heart: Years of high blood glucose may hurt the body's veins and nerves that post of the heart. That raises your opportunity of getting a heart condition. It can cause coronary disappointment early and respiratory failures or strokes later. High imperative signs and elevated cholesterol make the issues considerably almost certain.

Kidneys: Diabetes can influence veins in the kidneys, as well, all together that they probably won't work likewise. Following a couple of long periods of difficulty, they could get together.

Feet: High blood glucose can hurt blood stream and harm nerves, which may cause cuts, scratches, or wounds to recuperate gradually. A patient will lose some inclination in the feet, which shields from seeing wounds that will get tainted. On the off chance that a disease quits fooling around, it'd mean the patient might want to have a foot eliminated. Nerves: If high blood glucose harms the nerves, called diabetic neuropathy, it would potentially feel torment, shivering, or deadness, particularly in the feet.

Skin: Diabetes may make the patient bound to have yeast contaminations, tingling, or earthy colored or layered patches. Men with diabetes could likewise be in peril for sexual issues, since high blood glucose can hurt blood stream and harm nerves that the body must get and keep an erection [13].

\subsection{Factors That Reduce Diabetic Complications}

Diabetic inconveniences are regularly decreased by eating well-eating routine 
accomplishing a solid weight and doing exercise, going for ordinary exams, and consistency to the treatment is an imperative thing and all help to improve blood glucose control and can lessen the occasion of complexities of diabetes like retinopathy, hypertension, removals, coronary disappointment, and renal disappointment with numerous different difficulties [14].

Great propensities go an all-encompassing path toward forestalling the contrary medical issues that diabetes can cause. Keeping tight control of blood sugar, it's the most straightforward gratitude to maintain a strategic distance from diabetes complexities. Levels should remain in these solid ranges the greatest sum as could reasonably be expected, watch fundamental signs and cholesterol. If they're too high, it is more likely to get other health problem like heart disease. Endeavor to keep BP under 140/90 and absolute cholesterol at or under 200, regular exercise. It's the chief single measure that can prevent type 2 diabetes, diet and healthful control, regular exams. Blood, pee, and do different tests to recognize any issues. These visits are particularly significant since numerous diabetes inconveniences don't have clear admonition signs, avoid smoking, check feet daily. Look for any cuts, wounds, scratches, rankles, ingrown toenails, redness, or growing. Wash and dry feet cautiously consistently. Utilizing cream to avoid dry skin [15].

\subsection{Attitude and Practice of Pharmacological Glycemic Control}

Diabetic confusions like diabetic ketoacidosis, miniature, and full scale vascular diabetic intricacies, and their related antagonistic results are personally connected with problematic glycemic control in clinical practice. Each $1 \%$ decrease inside the mean glycated hemoglobin ( $\mathrm{HbAlc}$ ) has been demonstrated to be related to a markdown in danger of $21 \%$ for passing identified with diabetes, $14 \%$ for myocardial infarct, and $37 \%$ for miniature vascular difficulties [16].

Studies have demonstrated that a huge decrease in mortality and dismalness happens with improved glycemic control. This may spill out to a rebate in micro vascular entanglements like low foundational irritation, by the avoidance of safe brokenness and security of the endothelium and of the mitochondrial ultra structure and execution [17].

Regardless of the pathogenesis, uncontrolled diabetes or poor glycemic control is identified with constant hyperglycemia, bringing about the occasion of long haul miniature vascular, large scale vascular, and neuropathic confusions. The objective for long haul glycemic control in patients with diabetes is glycated hemoglobin A1c (HbA1c) estimation of however 7\% [18].

Most patients with type 2 diabetes neglect to direct hyperglycemia with diet and exercise alone and need pharmacotherapy. Glycemic control in diabetes is essential to stop long haul miniature and macro vascular inconveniences. The assortment of things besides glycated hemoglobin (HbAlc) level will impact treatment regimens, and practice rules underline the need for attending the treatment of other cardiovascular danger factors, like blood vessel hypertension and dyslipidemia [19]. 
The World Health Organization has demonstrated that adherence to long haul treatment for constant diseases in created nations midpoints just around half. Adherence rates are normally decreased for patients with constant conditions than those with intense conditions; this is frequently identified with the drawn-out nature of ongoing sicknesses because the decrease in adherence is generally fast after the essential half year of treatment. Such decreased adherence prompts chronic frailty results as well as highlights a critical effect on medical care costs. In this manner, the overall administration of diabetes should address adherence likewise as suitable medications [20].

\subsection{General Objectives}

The main objective of this study is to determine the Knowledge, Attitude and Practices (KAP) of common diabetic complications among diabetic patients at an Egyptian hospital in Mogadishu, Somali.

\section{Specific Objectives}

1) To determine knowledge of diabetic patients about diabetic complications among diabetic patients at an Egyptian hospital;

2) To understand factors that reduces the development of diabetic complications among diabetic patients at an Egyptian hospital;

3) To assess attitude and practice of pharmacological glycemic control among diabetic patients at an Egyptian hospital.

\section{Research Design}

The design of this was a descriptive qualitative study. This means that the sample was selected from the target and information was obtained respondents at the same time on a particular point in time. It's a descriptive design because it describes the knowledge, attitude, practice of common diabetes complications among diabetic patients at an Egyptian hospital in Mogadishu.

\subsection{Study Area}

Egyptian hospital in Mogadishu, located in Hodan district. The medical work of the Committee in Somalia started on 8/2011 as Abu Bakar Al-Siddiq clinics. Two months later, in early 9/2011, the Egyptian Hospital was established with good potential. The average number of operations per day reached about 20 and it was the only free hospital for nearly four years. Highly qualified consultant and specialists' doctors are operating in the Egyptian hospital.

\subsection{Study Population}

The study population was 98 patients affected with diabetes mellitus at an Egyptian hospital in Mogadishu which conducted in two weeks.

\subsection{Inclusion Criteria}

Diabetic patients who attending Egyptian hospitals responded to the research 
questions.

\subsection{Exclusion Criteria}

Patients attending Egyptian hospital who are not diabetic patients are excluded from the study of the research.

\subsection{Sample Size}

It was conducted from May up to July in 2020 A sample of 98 respondents with diabetes mellitus was taken in this study using non probability sampling. The sample size refers to the number of respondents to be selected from the target population.

\subsection{Sampling Procedure}

The non-probability consecutive sampling method was used to select diabetic patients at an Egyptian hospital.

\subsection{Research Instrument}

The questionnaire method was used for data collection of this research.

\subsection{Data Analysis}

After the collection of the data all interviewed questionnaires were checked for their completeness correctness and internal consistency to exclude missing or inconsistent data and those were discarded corrected date was entered into the computer the date will be analyzed by using the statistical software named Statistical Package for Social Science (SPSS 20).

\subsection{Ethical Considerations}

Every respondent was asked for permission to complete the questionnaire. A good explanation of the respondents was done before filling the questionnaire. Privacy and confidentiality were kept.

\subsection{Limitations of the Study}

The research faced a number of problems includes:

1) The study was limited to one hospital only;

2) Time constraints because the study was conducted under a short duration probably from May 2020 up to June 2020;

3) COVID-19 pandemic which limits meeting and intervention with patients.

\section{Result}

The majority of the respondents were male $58(59.18 \%)$ while 40 of the respondents were female (40.82\%), 23 (23.5\%) out of the 98 respondents were less than 20 years, $10(10.2 \%)$ out of the 98 respondents were between 20 and 39 years of age, $36(36.7 \%)$ out of the 98 respondents were between 40 and 60 years of age, 
and $29(29.6 \%)$ out of the 98 respondents were greater than 60 years of age. The majority of the respondents were married 60 (61.2\%) out of the 98 of the respondents, $28(28.6 \%)$ of the respondents were single, $4(4.1 \%)$ of them were divorced and the remaining $6(6.1 \%)$ of the respondents were widowed. The majority was illiterate $55.1 \%$ and elementary was $23 / 5 \%$ while secondary $16.3 \%$ and $5.1 \%$ were college level. $55.1 \%$ of the respondents were unemployed and this factor among many others might be responsible for the increasing complications of diabetes mellitus patients suffer because most of them don't enough expenses to monitor and buy quality medications also this alone might cause poor practice, knowledge and attitude of patients towards diabetes. $21.4 \%$ of the respondents were also students thus may be dependent on their parents for treatment and living. $22.5 \%$ of the respondents were employees and $1 \%$ was a freelancer (Table 1).

$26.53 \%$ of the respondents were having type 1 diabetes mellitus, majority of the respondents were having type 2 diabetes almost $47.96 \%$. The remaining $25.51 \%$ of the respondents don't know the type of diabetes they had. The majority of the respondents $82.65 \%$ were symptomatic while the remaining $17.35 \%$ mode of diagnosis was incidental. " $26.5 \%$ of the respondents their age of onset of diabetes was less than 20 years of age, while the majority $54.1 \%$ of the respondents their age of onset of diabetes was greater than 41 years of age Table 2".

Table 1. Socio demographic characteristics of respondents.

\begin{tabular}{|c|c|c|c|}
\hline Characteristic & & Frequency & Percent \% \\
\hline \multirow{2}{*}{ Gender } & Female & 58 & 59.2 \\
\hline & Male & 40 & 40.8 \\
\hline \multirow{4}{*}{ Age } & $>20$ years & 23 & 23.5 \\
\hline & $21-39$ & 10 & 10.2 \\
\hline & $40-60$ & 36 & 36.7 \\
\hline & $>61$ years & 29 & 29.6 \\
\hline \multirow{4}{*}{ Marital status } & Single & 28 & 28.6 \\
\hline & Married & 60 & 61.2 \\
\hline & Divorce & 4 & 4.1 \\
\hline & Widow & 6 & 6.1 \\
\hline \multirow{4}{*}{ Education level } & Illiterate & 54 & 55.1 \\
\hline & Elementary & 23 & 23.5 \\
\hline & Secondary & 16 & 16.3 \\
\hline & College & 5 & 5.1 \\
\hline \multirow{4}{*}{ Occupation } & Unemployment/housewife & 54 & 55.1 \\
\hline & Student & 21 & 21.4 \\
\hline & Employee & 22 & 22.5 \\
\hline & Free lancer & 1 & 1 \\
\hline
\end{tabular}


Majority of the respondents $44.9 \%$ had diabetes for duration of greater than 10 years. The respondent $31.6 \%$ had diabetes for duration between 5 to 10 years. $14.3 \%$ had diabetes for duration of 1 to 4 years and $9.2 \%$ only had it for a period of less than one year. Respondents by family history $53.1 \%$ of the respondents do not have a family history of diabetes while $46.9 \%$ of the respondents had a family history of diabetes (Table 2).

$60.2 \%$ of the respondents self-check diabetes which is a good practice while $39.8 \%$ do not self-check. $52 \%$ of the respondents control their diet while $48 \%$ do not control their diet. The majority of the respondents $63.3 \%$ do not do regular exercise, $36.7 \%$ do regular exercise. $54.1 \%$ of the respondents adhere to the treatment while $45.9 \%$ do not adhere to their treatment. $72.5 \%$ of the respondents know the common complications and $27.5 \%$ do not know. $41.8 \%$ of the respondents developed complications and 58.2\% does not develop any complications (Table 3).

The majority of respondents 9 (22\%) have Diabetic ketoacidosis as complications of diabetes mellitus while 8 (19.51\%) of respondents had foot ulcers and 6 (14.6) of the respondents had nephropathy (Table 4). The majority of the respondents $78.6 \%$ know factors that reduce the development of diabetic complications and $22.4 \%$ do not know " $37.8 \%$ of the respondents visited a doctor because of diabetic mellitus complications while $62.2 \%$ of the respondents never visited a doctor because of the complications Table 4".

\section{Discussion}

Diabetes is an important cause of morbidity and mortality all over the world.

Table 2. Response to knowledge questions.

\begin{tabular}{cccc}
\hline Variable & & Frequency & Percent \% \\
\hline \multirow{2}{*}{$\begin{array}{c}\text { The type of diabetes } \\
\text { respondents had }\end{array}$} & Type 1 & 26 & 26.53 \\
& Don't know & 47 & 47.96 \\
Mode of diagnosis & Incidental & 25 & 25.51 \\
& Symptomatic & 17 & 17.35 \\
& & 81 & 82.65 \\
Age at onset of diabetes & 21 years of age & 26 & 26.5 \\
& $31-40$ years of age & 6 & 6.1 \\
& 41 years of age & 13 & 13.3 \\
& & 53 & 54.1 \\
& $<1$ year & & \\
Duration of diabetes & 1 - 4 years & 9 & 9.2 \\
& 5 - 10 years & 14 & 14.3 \\
& $>10$ years & 31 & 31.6 \\
& & 44 & 44.9 \\
Family history of diabetes & Yes & 46 & 46.9 \\
& No & 52 & 53.1 \\
& & &
\end{tabular}


Table 3. Response to attitude and practice questions.

\begin{tabular}{cccc}
\hline Variable & & frequency & Percent \% \\
\hline Self-check of diabetes & Yes & 59 & 60.2 \\
& No & 39 & 39.8 \\
Do you control your diet & Yes & 51 & 52 \\
& No & 47 & 48 \\
Do you do regular exercise & Yes & 36 & 36.7 \\
No you adhere to your treatment & Yes & 62 & 63.3 \\
Do you know the common & No & 53 & 54.1 \\
complications of diabetes & Yes & 45 & 45.9 \\
Do you develop any complications of & no & 71 & 72.5 \\
diabetes mellitus & Yes & 27 & 27.5 \\
& No & 41 & 41.8 \\
\hline
\end{tabular}

Table 4. Response to complications of diabetes questions.

\begin{tabular}{cccc}
\hline Variable & & Frequency & Percent \% \\
\hline & Diabetic ketoacidosis & 9 & 22 \\
& Foot ulcer & 8 & 19.51 \\
& Retinopathy & 6 & 14.6 \\
Complications of diabetes & Nephropathy & 6 & 14.6 \\
mellitus you developed & Amputation & 4 & 9.8 \\
& Peripheral arterial disease & 1 & 2.43 \\
& Coronary artery disease & 1 & 2.43 \\
& Neuropathy & 1 & 2.43 \\
Do you know factors that reduce & Combined complications & 5 & 12.2 \\
development of diabetic complications & Yes & 77 & 78.6 \\
$\begin{array}{c}\text { Have you ever visited doctor because of } \\
\text { diabetic complication }\end{array}$ & No & 21 & 22.4 \\
& Yes & 37 & 37.8 \\
\hline
\end{tabular}

Because of lack of awareness about diabetes many patients with diabetes suffer from its complications. Studies from both developed and developing countries have reported that diabetes knowledge is generally poor among diabetic patients the patients in the present study had good general knowledge and had a good attitude and practice apart from regular exercise which was poor. However, it is difficult to compare our results with others, as most of the studies used different instruments and/or are carried out among different ethnic or age groups.

In the present study when looked for knowledge of complications of DM in the study population, we found Nephropathy, Foot ulcer and Retinopathy were the most known complication by the respondents. But Diabetic ketoacidosis was the most common complication (22\%). Other complications which respondents have developed are foot ulcer (19.51\%), nephropathy (14.6\%), and retinopathy 
(14.6\%). So the present study population had good knowledge of complications which may be because of long standing disease.

Conducting a study in the Uganda population which aimed to determine the knowledge of diabetic complications among diabetic malignant, they found that the most common diabetic complication known by DM patients was a diabetic foot (51.5\%), followed by hypertension (35.4\%), neuropathy (29.2\%), hypoactive sexual arousal (25.4\%), arousal disorder (21.5\%), retinopathy (17.7\%), heart disease (9.2\%), and nephropathy (5.4\%). The authors of the Ghana study also looked for an association between levels of knowledge of DM complication and socio-demographic characteristics. They reported that the elderly had adequate knowledge on diabetic complications than the very younger population with approximately $66.7 \%$ of participants with $16-20$ years duration of diabetes had adequate knowledge on diabetic complications than to those with 11 - 15-year duration (37.5\%), 5 - 10 year (19.2\%) and below 5 years (8.6\%). This can again be explained by the long-standing disease. The attitude and practices about the life style modifications in diabetics in the present study are as follows. Regular checkup was done by $60.2 \%$ of respondents, while $54.1 \%$ have adhered to treatment. Fifty-two percent of respondents were following diet schedule and only $36.7 \%$ did regular exercise. Some studies report a significant positive correlation between knowledge, attitude and practice. Better knowledge is associated with a better attitude and practices towards diabetes.

In a Bangladesh study done by Fatema et al. Respondents were aware of the major causes of diabetes half of the participants had knowledge regarding the components of a well-balanced diet. $76 \%$ are aware of the treatment of the diabetes. $70 \%$ of the study respondents reported that exercise helps in controlling the diabetes. The study mentioned that these differences in positive attitudes towards the treatment of DM are explained by socio demographic status as well.

\section{Conclusions}

We conclude that the KAP of DM patients was good regarding knowledge of diabetic complications. These findings may be due to the long-standing disease of patients which makes them learn things on their own. While only a few of them knew vasculopathy and heart diseases.

However, the study showed low levels of attitudes and practice towards regular exercise, also attitudes and practice toward control diet and treatment adherence were not satisfactory.

In general, this level of KAP is not optimal and needs more evaluation about the barriers of KAP in Somalia.

\section{Recommendations}

Knowledge, attitude and practice are essential or very important in preventing and controlling the development of diabetic complications. So to improve KAP the following is needed: 
- A comprehensive nationwide diabetic education program is necessary to improve this situation.

- Education and counseling about all the aspects of diabetes are needed.

- Group education, as well as individualized education programs, should be planned which can lead to better preventive and management techniques in diabetes.

- A multidisciplinary approach including a well-trained community doctor, diabetic and endocrinologist specialist, dietician, diabetic nurse and diabetic care unit.

\section{Conflicts of Interest}

The authors declare no conflicts of interest regarding the publication of this paper.

\section{References}

[1] Abejew, A.A., Belay, A.Z. and Kerie, M.W. (2015) Diabetic Complications among Adult Diabetic Patients of a Tertiary Hospital in Northeast Ethiopia. Advances in Public Health, 2015, Article ID: 290920. https://doi.org/10.1155/2015/290920

[2] Al-Asbali, T., Aldawari, S.A., Alzahim, I.A., Alalawi, H., Khandekar, R. and Lotfy, N.M. (2020) Knowledge, Attitude and Practice Regarding Diabetic Retinopathy Screening and Its Management among Diabetic Patients at a Private Hospital of Riyadh, Saudi Arabia. Saudi Journal of Ophthalmology, 34, 85-93. https://doi.org/10.4103/1319-4534.305040

[3] Al-Moosa, S., Allin, S., Jemiai, N., Al-Lawati, J. and Mossialos, E. (2006) Diabetes and Urbanization in the Omani Population: An Analysis of National Survey Data. Population Health Metrics, 4, Article No. 5. https://doi.org/10.1186/1478-7954-4-5

[4] Alsunni, A.A., Albaker, W.I., Almansour, A.H., Alenazi, A.S., Alaftan, M.S. and Badar, A. (2020) Knowledge, Attitude and Practice Regarding Ramadan Fasting and Related Determinants in Patients with Type 2 Diabetes at a Saudi Diabetes Clinic. Diabetes, Metabolic Syndrome and Obesity: Targets and Therapy, 13, 151-159. https://doi.org/10.2147/DMSO.S241496

[5] Alzaidi, M.M., Saeedalmalki, H. and Alghamdi, G.M. (2016) Assessing the Knowledge, Attitudes and Practice of Diabetic Complications among Physicians in Taif, Saudi Arabia. The Egyptian Journal of Hospital Medicine, 65, 693-695. https://doi.org/10.12816/0033784

[6] Aqeel, T. (2017) A Cross-Sectional Study Assessing Knowledge Attitude and Practice of Diabetic Patients at Tertiary Care Hospitals of Twin of Pakistan. Journal of Applied Pharmacy, 8, Article ID: 1000210J.

[7] Badruddin, N., Basit, A., Hydrie, M.Z.I. and Hakeem, R. (2002) Knowledge, Attitude and Practices of Patients Visiting a Diabetes Care Unit. Pakistan Journal of Nutrition, 1, 99-102. https://doi.org/10.3923/pin.2002.99.102

[8] Beiranvand, S., Fayazi, S. and Asadizaker, M. (2015) Effect of Educational Programs on the Knowledge, Attitude, and Practice of Foot Care in Patients with Diabetes. Jundishapur Journal of Chronic Disease Care, 4, e26540.

https://doi.org/10.5812/jjcdc.26540

[9] Bhatia, S., Singh, D., Shankar, P., Tutu, S., Kumar, A. and Dixit, R.K. (2015) Study of Knowledge, Attitude and Practice of General Population of Lucknow towards 
Hypertension. World Journal Pharmacy and Pharmaceutical Science, 4, 1674-1679.

[10] Delavari, A., Alikhani, S., Nili, S., Birjandi, R.H. and Birjandi, F. (2009) Quality of Care of Diabetes Mellitus Type II Patients in Iran. Archives of Iranian Medicine, 12, 492-495.

[11] Durand, W.F. (1935) Influence of Principal Factors on Performance. Aerodynamic Theory, January 2001, 315-324. https://doi.org/10.1007/978-3-642-91488-1 20

[12] Gautam, A., Bhatta, D.N. and Aryal, R.U. (2015) Diabetes Related Health Knowledge, Attitude and Practice among Diabetic Patients in Nepal. BMC Endocrine Disorders, 15, 25. https://doi.org/10.1186/s12902-015-0021-6

[13] Jacques, V., Mauriac, A.T., Leopold, A.B., Sabi, A.K., Ubald, A.-G., Vénérand, A. and François, D. (2016) Knowledge Attitude and Practice of General Physicians for Early Detection of Diabetic Nephropathy in Cotonou. Open Journal of Nephrology, 6, 122-131. https://doi.org/10.4236/ojneph.2016.64015

[14] Kadirvelu, A. (2012) Reality vs Illusion: Knowledge, Attitude and Practice among Diabetic Patients. International Journal of Collaborative Research on Internal Medicine \& Public Health, 4, 723-732.

[15] Kant, R. and Thapliyal, V. (2015) Knowledge Attitude and Practice of Type 2 Diabetic Patients in a Tertiary Care Teaching Hospital in India. Integrative Food, $\mathrm{Nu}$ trition and Metabolism, 2, 131-135. https://doi.org/10.15761/IFNM.1000115

[16] Mohammadi, S. (2015) Knowledge, Attitude and Practices on Diabetes among Type 2 Diabetic Patients in Iran: A Cross-Sectional Study. Science Journal of Public Health, 3, 520. https://doi.org/10.11648/j.sjph.20150304.20

[17] Obirikorang, Y., Obirikorang, C., Anto, E.O., Acheampong, E., Batu, E.N., Stella, A.D., Constance, O. and Brenya, P.K. (2016) Knowledge of Complications of Diabetes Mellitus among Patients Visiting the Diabetes Clinic at Sampa Government Hospital, Ghana: A Descriptive Study. BMC Public Health, 16, Article No. 637. https://doi.org/10.1186/s12889-016-3311-7

[18] Palaian, S., Acharya, L.D., Rao, P.G.M., Shankar, P.R., Nair, N.M. and Nair, N.P. (2006) Knowledge, Attitude, and Practice Outcomes: Evaluating the Impact of Counseling in Hospitalized Diabetic Patients in India. $P$ and T, 31, 383-392.

[19] Rahaman, K.S., Majdzadeh, R., Naieni, K.H. and Raza, O. (2017) Knowledge, Attitude and Practices (KAP) Regarding Chronic Complications of Diabetes among Patients with Type 2 Diabetes in Dhaka. International Journal of Endocrinology and Metabolism, 15, e12555. https://doi.org/10.5812/ijem.12555

[20] Srinivasan, N.K., John, D., Rebekah, G., Kujur, E.S., Paul, P. and John, S.S. (2017) Diabetes and Diabetic Retinopathy: Knowledge, Attitude, Practice (KAP) among Diabetic Patients in a Tertiary Eye Care Centre. Journal of Clinical and Diagnostic Research, 11, NC01-NC07. https://doi.org/10.7860/JCDR/2017/27027.10174 